\title{
Gamma-ray burst host galaxies and the link to star-formation
}

\author{
J. P. U. Fynbo*, J. Hjorth, D. Malesani, J. Sollerman, D. Watson \\ Dark Cosmology Centre, Copenhagen University \\ Copenhagen O,DK-2100, Denmark \\ *E-mail:jfynbo@dark-cosmology.dk \\ www.dark-cosmology.dk \\ P. Jakobsson \\ Centre for Astrophysics Research, University of Hertfordshire \\ CollegeLane, Hatfield, Herts, AL109AB, UK \\ J. Gorosabel \\ Instituto de Astrofísica de Andalucía (CSIC), Apartado de Correos 3004, 18080 Granada, Spain \\ A. O. Jaunsen \\ Institute of Theoretical Astrophysics, PO Box 1029 Blindern, 0315 Oslo, Norway
}

\begin{abstract}
We briefly review the current status of the study of long-duration gamma-ray burst (GRB) host galaxies. GRB host galaxies are mainly interesting to study for two reasons: 1) they may help us understand where and when massive stars were formed throughout cosmic history, and 2) the properties of host galaxies and the localization within the hosts where GRBs are formed may give essential clues to the precise nature of the progenitors. The main current problem is to understand to what degree GRBs are biased tracers of star formation. If GRBs are only formed by low-metallicity stars, then their host galaxies will not give a representative view of where stars are formed in the Universe (at least not a low redshifts). On the other hand, if there is no dependency on metallicity then the nature of the host galaxies leads to the perhaps surprising conclusion that most stars are formed in dwarf galaxies. In order to resolve this issue and to fully exploit the potential of GRBs as probes of star-forming galaxies throughout the observable universe it is mandatory that a complete sample of bursts with redshifts and host galaxy detections is built.
\end{abstract}

Keywords: Style file; LATEX; Proceedings; World Scientific Publishing.

\section{Introduction}

The association between long-duration $\left(\mathrm{T}_{90}>2 \mathrm{~s}^{1}\right)$ GRBs and massive stars and hence the link between GRBs and on-going star-formation found its first empirical basis with the detection of the first host galaxies (e.g. ${ }^{2}$ ). Subsequently, the evidence was further strengthened with the discovery of supernovae $(\mathrm{SNe})$ associated with $\mathrm{GRBs}^{3-9}$ (for a recent review of the GRB/SN association see ${ }^{11}$ ).

Because of the link between long-duration GRBs and massive stars and due to the fact that GRBs can be detected from both the most distant and the most dust obscured regions in the universe GRBs were quickly identified to be very promising tracers of star-formation (e.g. ${ }^{12}$ ). However, this potential has so far not really resulted in an improved census of the star-formation activity due to complications discussed in the next section. 
A major issue currently under discussion is if GRBs are unbiased tracers of star formation. More precisely, it is not clear if GRBs are caused by the same (small) fraction of all dying massive stars (unbiased tracers), or if GRBs only trace a limited segment defined by parameters such as, e.g., metallicity or circumstellar density (biased tracers).

The currently operating Swift satellite ${ }^{22}$ has revolutionized GRB research with its frequent, rapid, and precise localization of GRBs. Now it is for the first time possible in practice to use GRBs as powerful probes. It is mandatory that this potential is exploited while Swift is still operating.

\section{Complications in the use of GRBs as SF tracers}

\subsection{The contamination from chance projections}

The first important question to ask is: are GRB host galaxies operationally welldefined as a class? In terms of an operational definition the case is not so clear. If we define the host galaxy of a particular burst to be the galaxy nearest to the lineof-sight, we need to worry about chance projection. ${ }^{13}$ In the majority of cases where an optical afterglow has been detected and localized with subarcsecond accuracy and where the field has been observed to deep limits a galaxy has been detected within an impact parameter less than 1 arcsec (e.g. ${ }^{14}$ and Fig. 1). The probability for this to happen by chance is typically found to be of the order $10^{-3}$ or less. For a sample of a few hundreds GRBs chance projection should hence not be a serious concern.

When the redshift of the GRB is measured, the association can of course be proven beyond doubt by measuring also the redshift of the potential host. However, the fraction of GRBs for which the redshift is measured from the afterglow is still only $\sim 50 \%^{17}$ so the association cannot be made in this way for all GRBs.

In any case, there are misidentifications in the literature (see ${ }^{15}$ for two examples). Other noteworthy examples are the cases of GRB 030429 and GRB 041006: In the field of GRB 030429 a galaxy was detected at an impact parameter of $1.2 \operatorname{arcsec}$ from the position of the afterglow and no galaxy was detected at the position of the afterglow to deep limits. However, spectroscopy of the afterglow and of the nearby galaxy established that the two were unrelated. $^{18}$ In the case of GRB 041006 a galaxy 1.0 arcsec from the afterglow position was suggested to be the host, ${ }^{19}$ but later $H S T$ imaging revealed another galaxy at the afterglow position. ${ }^{20}$

In conclusion, for GRBs localized with an accuracy worse than about 1 arcsec chance projection cannot be excluded, but chance projection is not a major obstacle for the use of GRBs to select star-forming galaxies.

\subsection{Dark bursts and incomplete samples}

A crucial issue when using GRBs (or any other class of tracer) to probe starformation is sample selection. Whereas the detection of the GRB itself poses no 


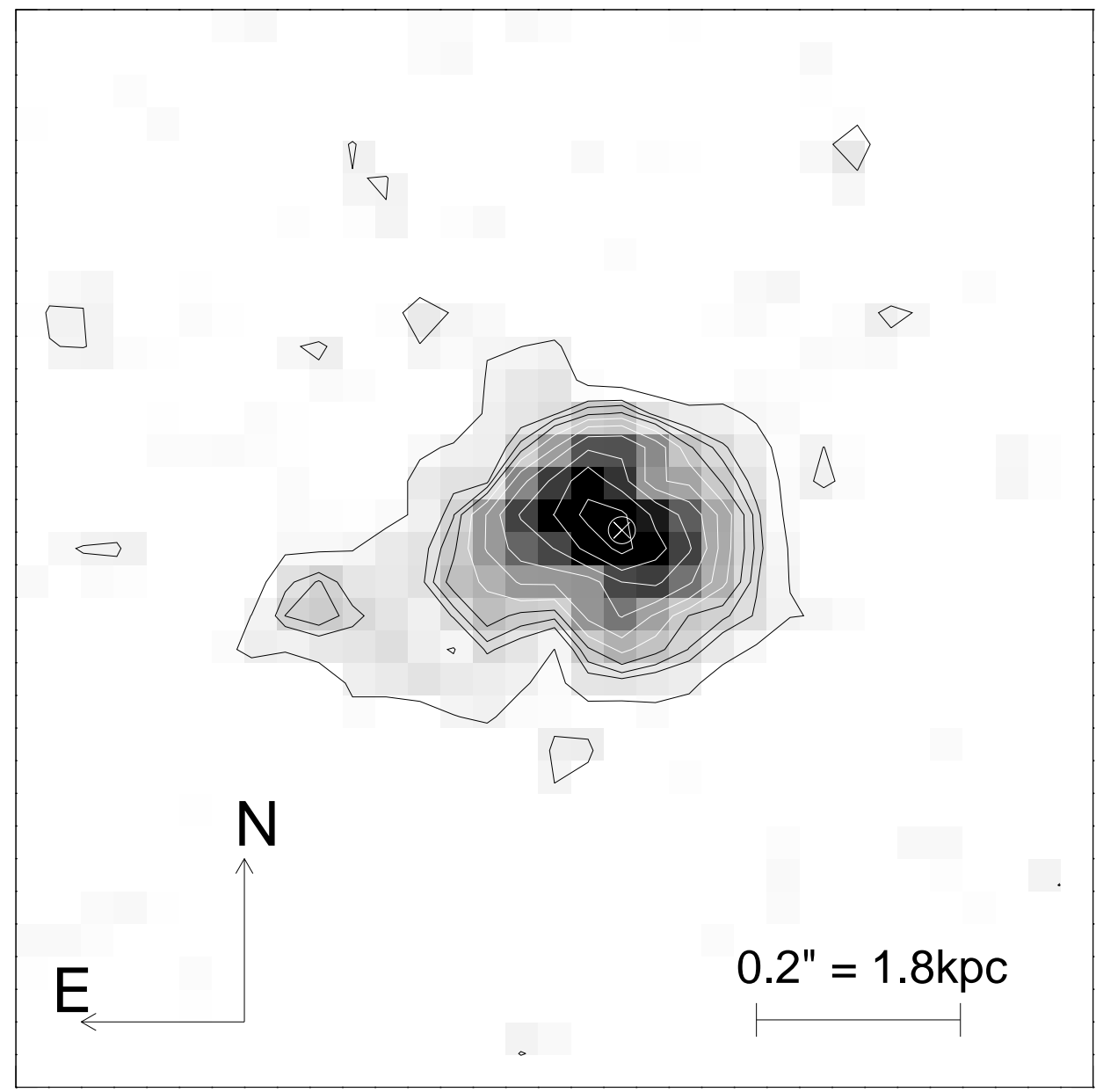

Fig. 1. The $1 \times 1 \operatorname{arcsec}^{2}$ field around the host galaxy of the $z=2.33$ HETE-II GRB 021004 observed with the HST $\left(\right.$ from $\left.^{16}\right)$. The GRB went off near the center of the galaxy. The position of the GRB is marked with a cross and an error circle and in coincides with the centroid of the galaxy to within a few hundredths of an arcsec. In cases like this there is no problem in identifying the correct host galaxy.

bias against dust obscured star-formation this is not the case for the softer afterglow emission which is crucial for obtaining the precise localization as well as measuring redshifts (see, e.g., ${ }^{21}$ and the contribution from Dr. Fiore in these proceedings).

In the samples of GRBs detected with satellites prior to the currently operating Swift satellite ${ }^{22}$ the fraction of GRBs with detected optical afterglows was only about $30 \% .{ }^{23,24}$ Much of this incompleteness was caused by random factors such as weather or unfortunate celestial positions of the bursts, but some remained undetected despite both early and deep limits. It is possible that some of these so called "dark bursts" could be caused by GRBs in very dusty environments ${ }^{25}$ and hence the 
sample of GRBs with detected optical afterglows could very well be systematically biased against dust obscured star formation (see also ${ }^{26,27}$ for recent discussions of dark bursts).

In any case, such a high incompleteness imposes a large uncertainty on statistical studies based on GRB host galaxies derived from these early missions.

Due to the much more precise and rapid localization capability of Swift it is now possible to build much more complete samples (see below).

\subsection{Are some long GRBs not associated with massive stellar death?}

Recently, it has been found that some long-duration GRBs are not associated with SNe, namely GRB $060505^{28}$ and GRB 060614. ${ }^{28,30,31}$ This means that either some massive stars die without producing even faint supernovae ${ }^{28,30}$ or, alternatively, some long-duration GRBs are caused by other mechanisms than collapsing massive stars. ${ }^{31,32}$

At least for the case of GRB 060505 the evidence points to the former $\left(\mathrm{see}^{28,29}\right)$. However, if some long-duration GRBs indeed are caused by other progenitors than massive stars then a new classification that can distinguish between long GRBs from massive stars and those from other mechanisms is required. So far no such scheme has been found $\left(\right.$ see $\left.^{33}\right)$ 国

\section{What do GRB host galaxies tell us about star formation despite the complications?}

\subsection{Early results}

Despite the complications mentioned above, the previous decade of GRB host galaxy studies has after all taught us a lot about GRBs and star formation (see ${ }^{40,41}$ for early reviews). GRB hosts were early on found to be predominantly faint, blue starforming galaxies. ${ }^{2}$ The early studies found that these properties of GRB hosts were consistent with the expectation if GRBs are unbiased tracers of star-formation. ${ }^{2,42}$ It was also realized early on that GRBs offer a unique possibility to locate and study star-formation activity in dwarf galaxies at $z>2 .{ }^{43}$ This is basically impossible with any other currently existing method. The star-formation rates were found to be modest, but the specific star-formation rates are among the highest ever found. ${ }^{44}$ GRB hosts are hence typically in a starburst state.

\subsection{Current status}

As the samples have grown larger it has become clear that GRB hosts in general have low luminosities. As an example, not a single GRB at $z>2.5$ has a host

${ }^{a}$ A classification scheme has been proposed, ${ }^{34}$ but this scheme is ambiguous and not operational (e.g., for GRB 060505), involves observables that are not available for most bursts (the associated supernova), and the nature of the progenitor, which is not observable at all. 


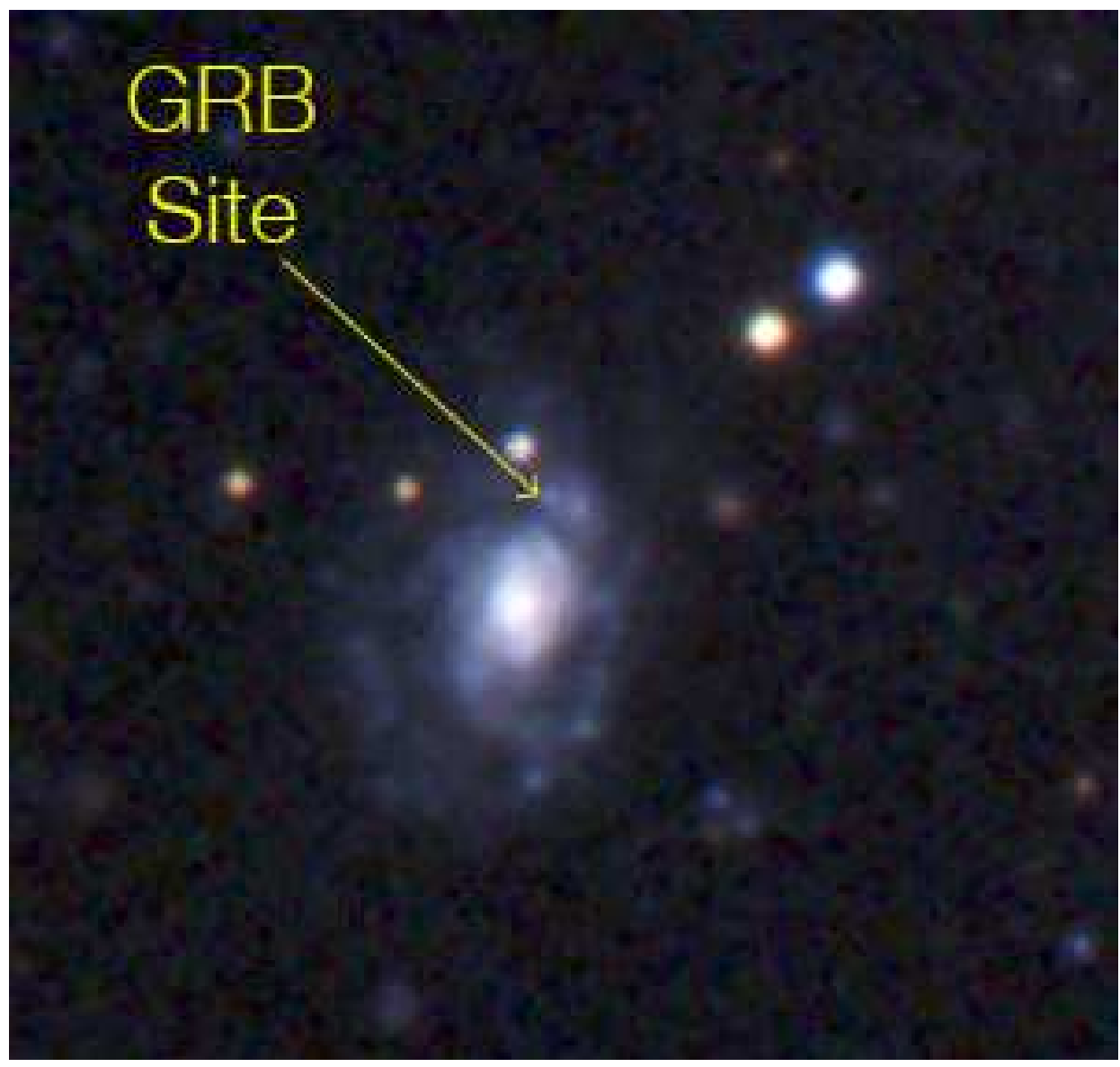

Fig. 2. The host galaxy of GRB 060505 as observed with the ESO VLT ${ }^{29}$ (see also. ${ }^{32}$ This SNless long GRB occurred in a star-forming region in one of the spiral arms of a late-type host. This is strong evidence that the progenitor was a massive star. The properties of this host is also within the range found for other long GRBs. As an example, the host galaxy of GRB 050824 is very similar in terms of luminosity and star-formation rate ${ }^{35}$ and the location within the host is similar to the location of other long GRBs in spiral hosts. ${ }^{36-39}$

galaxy that is bright enough that it would be detected in the ground based surveys for Lyman-break galaxies at similar redshifts (i.e. $\mathrm{R}<25.5$ ). This implies that either the Lyman-break galaxies are just the tip of an iceberg of star-forming galaxies at $z>2.5$ or GRB are biased towards faint hosts. Also nearby GRB hosts are found to be of low luminosity. ${ }^{45,46}$

The environments of GRB host galaxies has not been subject of many studies. At low redshifts ${ }^{47}$ studied the field of the host galaxy of GRB 980425, that was reported to be member of a group. However, based on redshift measurements of the proposed group members ${ }^{47}$ could establish that the host of GRB 980425 is 
an isolated dwarf galaxy. The host galaxy of GRB 030329 was found to have a companion at projected distance of $600 \mathrm{kpc} .{ }^{48}$ The host galaxy of GRB 060505 has been located to the outskirts of a filament extending from the galaxy cluster Abell $3837 .{ }^{29}$ At redshifts $z \gtrsim 2$ a few GRB fields have been studied using narrow band Ly $\alpha$ imaging. ${ }^{4-51}$ In all cases several other galaxies at the same redshift as the GRB host were identified, but it is not sure whether the galaxy densities in these fields are higher than in blank fields as no blank field studies have been carried out at similar redshifts. However, the density of Ly $\alpha$ emitters were found to be as high as in the fields around powerful radio sources that have been proposed to be forming protoclusters, which would suggest that GRBs could reside in overdense fields at $z \gtrsim 2$. Another study ${ }^{52}$ argue for a low galaxy density in GRB host galaxy environments, but this study is based on data probing very small fields-of-view around the hosts.

The absence of bright dust-obscured hosts in the GRB host samples (but see also $^{53}$ ) has also been argued to be inconsistent with a scenario where GRBs are unbiased tracers, ${ }^{54,55}$ but, as it has recently been pointed out, "there is sufficient uncertainty in models and underlying assumptions, as yet poorly constrained by observation (e.g. the adopted dust temperature) that a correlation between massive, dust-enshrouded star formation and GRB production cannot be firmly ruled out."

In addition, GRB hosts have been found to be more frequent Ly $\alpha$ emitters than Lyman-break galaxies at similar redshifts, which could be due to a low metallicity preference. ${ }^{50}$ However, all of these studies targeted very incomplete pre-Swift samples and this raises the question whether the faint hosts can be explained by, e.g., a bias against dust obscured GRB afterglows.

A very important result is that GRBs and core-collapse SNe are found in different environments. ${ }^{57}$ The same study also found that GRB host galaxies at $z<1$ are fainter than the host galaxies of core-collapse SNe. This study is also based on incomplete pre-Swift samples, but as the SNe samples are also biased against dusty regions this result does seem to be strong evidence that GRBs are biased towards massive stars with low metallicity ( based on nearly the same data as in ${ }^{57}$ has, however, concluded that "GRB hosts trace the starburst population at high redshift, as similarly concentrated galaxies at $z>2$ are undergoing a disproportionate amount of star formation for their luminosities. Furthermore, our results show that GRBs are not only an effective tracer of star formation but are perhaps ideal tracers of typical galaxies undergoing star formation at any epoch, making them perhaps our best hope of locating the earliest galaxies at $z>7 " .{ }^{60}$ A similar conclusion has been reached in other studies. ${ }^{61,62}$

A recent study of a sample of Swift GRBs have found that Swift GRBs appear to have fainter hosts than pre-Swift GRBs, ${ }^{63}$ presumably due their larger distance. ${ }^{17}$

\section{Progenitor models}

GRB progenitor models also give some indication of whether GRBs will be biased tracers. In the collapsar models for a GRB the central ingredients are formation of 
a rapidly rotating black hole and removal of the Hydrogen/Helium envelope before the explosion $\left(\mathrm{see}^{11,64}\right)$. The need for rapid rotation suggests that the progenitors need to have a low metallicity stellar wind, in order to avoid removing angular momentum from the star. On the other hand, the need to remove the Hydrogen envelope suggests that a high metallicity is needed at least for some single star progenitor models (e.g. ${ }^{65}$ their Fig. 7. See also ${ }^{66}$ ). Binary progenitor models have also been explored. ${ }^{66}$ We note that low metallicity massive stars in the Magellanic clouds do not appear to rotate faster than massive stars in the Milky Way despite their lower metallicities. ${ }^{67}$ There is also evidence that some GRBs are not related to the formation of a black hole, but rather a neutron star. ${ }^{10,69}$ There is also evidence for sustained activity of the inner engine. ${ }^{35,70}$ Whether the currently favored models are consistent with this evidence is unclear to the authors. Therefore, there is still some open issues concerning the role of metallicity in GRB progenitor models. There are other models for long duration GRBs where there is no obvious dependency on metallicity. ${ }^{68,71}$

\section{Outlook}

A number of steps need to be taken before the potential of GRB host galaxies as probes of the cosmic star-formation activity can really be exploited.

\subsection{Building a complete sample}

A crucial obstacle to overcome is to collect a complete sample and to secure as high a redshift completeness as possible. As pointed out by Dr. Jakobsson in these proceedings, efforts are ongoing to this end. The sample includes only Swift bursts fulfilling:

- $\mathrm{T}_{90}>2 \mathrm{~s}$.

- A rapidly distributed XRT error circle.

- Low foreground Galactic extinction: $\mathrm{A}_{\mathrm{V}}<0.5 \mathrm{mag}$.

- Declinations within $[-70,70]$ degrees.

We are currently working on detecting the hosts and measuring redshifts for as many of these bursts as possible. By December 2006, there is a total of 85 of these bursts, 43 with spectroscopic redshifts measurements (with a mean value of $\left.z_{\text {med }}=2.41\right), 22$ with no afterglow detection, and 20 with optical and/or IR afterglow detection but unknown redshift either because no spectrum was secured or no lines were detected on which to base a redshift.

\subsection{Getting Dark bursts into the picture}

Another crucial obstacle is to tackle the question of dark bursts. In Fig. 3 we show an updated version of the dark burst figure from. ${ }^{26}$ For Swift GRBs at least $25 \%$ 
are dark $\mathrm{b}$. An important step forward has been taken with the refined astrometry purely based on the X-ray afterglows. ${ }^{76}$ Now a substantial fraction of GRBs only detected in the X-rays have astrometry accurate to be better than 2 arcsec. This still leaves some probability for chance alignment, but it is now possible to make statistical comparison of X-ray based error-circles for dark bursts and bursts that are not dark and look, e.g., for a higher density of dusty galaxies in the dark burst error circles.

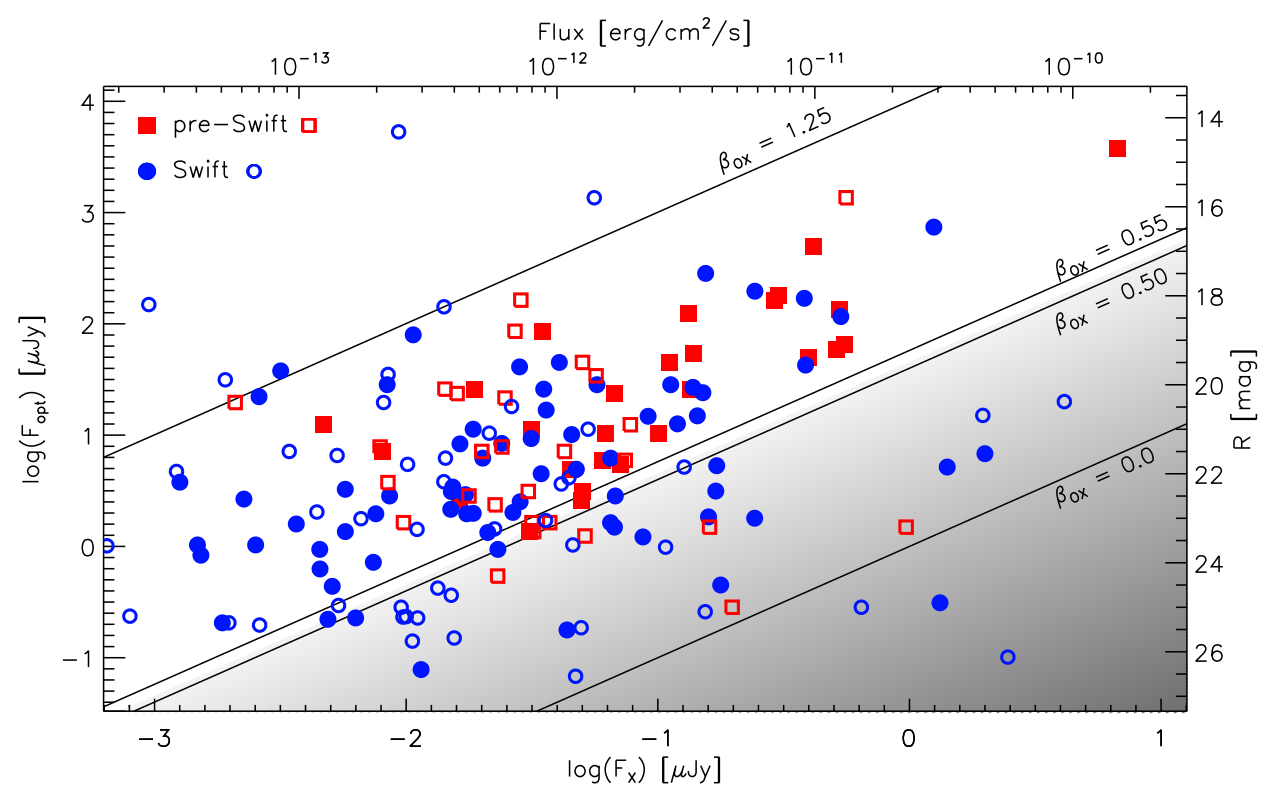

Fig. 3. A diagram of optical flux vs. X-ray flux for all long-duration bursts with available data (until 1 Jan 2007). The magnitudes have been corrected for Galactic extinction. Filled symbols indicate optical detections while open symbols are upper limits. Lines of constant optical-to-Xray spectral slope $\beta_{\mathrm{OX}}$ are shown along with the corresponding value. We define dark bursts as those which have $\beta_{\mathrm{OX}}<0.5$. The Swift dark bursts fraction is around $25 \%$ compared to the pre-Swift value of $10 \%$. Note that the majority of the Swift X-ray fluxes have been obtained from GCNs which only give an approximate value. Adapted from. ${ }^{26}$

\subsection{Simulations}

Finally, there is a need for more predictions of which properties we expect of GRB host galaxies under different assumptions about the progenitors of GRBs. Could

\footnotetext{
${ }^{\mathrm{b}}$ Note that a dark burst here is defined as a burst with an X-ray to optical slope too shallow for the synchrotron fireball model. Hence, according to this definition, a burst with no detected optical afterglow is not necessarily a dark burst, and some dark bursts have detected optical afterglows (GRB 050401 is an example ${ }^{75}$ ).
} 
the fact that most GRB hosts are so faint simply be telling us that this is the type of galaxies responsible for the majority of the star-formation activity? At redshifts $z \gtrsim 2$ the faint end slope for star-forming galaxies has been found to be very steep so this is not an unreasonable suggestion. ${ }^{49,51}$ There has so far only been a few such studies trying to predict the properties of GRB hosts (e.g. ${ }^{77-79}$ ), but much more work is needed (e.g. using larger simulated volumes and exploring different recipes for including star-formation in the simulations).

\section{References}

\section{References}

1. C. Kouveliotou et al., ApJ 413, L101 (1993).

2. D. W. Hogg \& Fruchter A. S., ApJ 520, 54 (1999).

3. T. Galama et al. Nature 395, 670 (1998).

4. J. Hjorth et al. Nature 423, 847 (2003).

5. K. Stanek et al. ApJL 591, L17 (2003).

6. T. Matheson et al. ApJ 599, 394 (2003).

7. J. Sollerman et al., $A \mathscr{G} A$ 454, 503 (2006).

8. M. Modjaz et al., ApJL 645, L21 (2006).

9. E. Pian et al., Nature 442, 1011 (2006).

10. K. Maeda et al., ApJL 658, L5 (2007).

11. S. E. Woosley \& J. S. Bloom, ARA $\& A$ 44, 507 (2006).

12. R. A. M. J. Wijers et al., MNRAS 294, L13 (1998).

13. D. L. Band \& D. H. Hartmann, ApJ 493, 555 (1998).

14. J. S. Bloom et al., $A J$ 123, 1111 (2002).

15. A. O. Jaunsen et al., $A \& A$ 402, 125 (2003).

16. J. P. U. Fynbo et al., ApJ 633, 317 (2005).

17. P. Jakobsson et al., $A \mathscr{E} A$ 447, 897 (2006).

18. P. Jakobsson et al., $A \xi A$ 427, 785 (2004).

19. S. Covino et al., GCN Circ. 2803 (2004).

20. A. M. Soderberg et al., ApJ, 636, 391 (2006).

21. F. Fiore et al., astro-ph/0610740 (2006).

22. N. Gehrels et al., ApJ 611, 1005 (2004).

23. J. P. U. Fynbo et al., A $\& A$ 369, 373 (2001).

24. D. Lazzati et al., MNRAS 330, 583 (2002).

25. P. Groot et al., ApJ 493, L27 (1998).

26. P. Jakobsson et al., ApJ 617, L21 (2004).

27. E. Rol et al., ApJ 624, 868 (2005)

28. J. P. U. Fynbo et al., Nature 444, 1047 (2006).

29. C. C. Thöne et al. ApJ submitted, astro-ph/0703407 (2007)

30. M. Della Valle et al., Nature 444, 1050 (2006).

31. A. Gal-Yam et al., Nature 444, 1053 (2006).

32. E. O. Ofek et al., ApJ submitted, astro-ph/0703192 (2007).

33. N. Gehrels et al., Nature 444, 1044 (2006).

34. B. Zhang et al., ApJL 655, L25 (2007).

35. J. Sollerman et al., $A \mathscr{G} A$ in press, astro-ph/0701736 (2007).

36. J. P. U. Fynbo et al., ApJL 542, L89 (2000).

37. J. Sollerman et al., $A \mathscr{E} A$ 386, 944 (2002).

38. E. Le Floc'h et al., ApJL 581, L81 (2002). 
39. P. Jakobsson et al., ApJ 629, 45 (2005).

40. J. van Paradijs et al., ARA\&A, 38, 379 (2000).

41. G. Djorgovski et al., Proc. SPIE, vol. 4834 (2003).

42. S. Mao \& Mo, H. J. ApJ 339, L1 (1998).

43. B. L. Jensen et al., $A \& A$ 370, 909 (2001).

44. L. Christensen et al., $A \& A$ 425, 913 (2004)

45. J. Sollerman et al., NewA 11, 103 (2005).

46. K. Z. Stanek et al., AcA 56, 333 (2006).

47. S. Foely et al., $A \mathscr{E} A$ 447, 891 (2005).

48. J. Gorosabel et al., $A \mathscr{E} A$ 444, 711 (2005).

49. J. P. U. Fynbo et al., $A \& A$ 388, 425 (2002).

50. J. P. U. Fynbo et al. $A \& A$ 406, L63 (2003).

51. P. Jakobsson et al., MNRAS 362, 465 (2005).

52. C. G. Bornancini et al., Apj 614, 84 (2004).

53. E. Berger et al., ApJ 588, 99 (2003).

54. E. Le Floc'h et al., $A \mathscr{E} A$ 400, 499 (2003).

55. N. R. Tanvir et al., MNRAS 352, 1073 (2004).

56. R. S. Priddey et al. MNRAS 369, 1189 (2006).

57. A. S. Fruchter et al. Nature 441, 463 (2006).

58. C. Wolf \& P. Podsiadlowski, MNRAS 375, 1049 (2007).

59. M. Modjaz et al., AJ, submitted, astro-ph/0701246 (2007)

60. C. Conselice et al., ApJ 633, 29 (2005).

61. J. P. U. Fynbo et al., $A \mathscr{E} A L$ 451, L47 (2006).

62. A. R. Velasco et al., ApJ, submitted (2007).

63. J. E. Ovaldsen et al., ApJ inpress, astro-ph/0703388 (2007).

64. C. Fryer et al., $A p$, submitted, astro-ph/0702338 (2007).

65. A. Heger et al., ApJ 591, 288 (2003).

66. C. L. Fryer \& A. Heger, ApJ 623, 302 (2005).

67. L. R. Penny et al., ApJ 617, 1316 (2004).

68. R. Ouyed et al., ApJ 632, 1001 (2005).

69. P. Mazzali et al., Nature 442, 1018 (2006).

70. D. Grupe et al., ApJ, in press, astro-ph/0611240 (2006).

71. A. Dar \& A. de Rújula Physics Reports 405, 203 (2004).

72. P. Jakobsson et al., $A \& \& A L$ 460, L13 (2006).

73. N. Kawai et al., Nature 440, 184 (2006).

74. R. L. C. Starling et al., A $₫ A$ 442, L21 (2006).

75. D. Watson et al., ApJ 652, 1011 (2006).

76. N. R. Butler $A J$ 133, 1027 (2007).

77. S. Courty et al., MNRAS 354, 581 (2004).

78. S. Courty et al., MNRAS, in press, astro-ph/0701762 (2007).

79. S. E. Nuza et al., MNRAS 375, 665 (2007). 